\title{
Global Myocardial Work Is Superior to Global Longitudinal Strain to Predict Significant Coronary Artery Disease in Patients With Normal Left Ventricular Function and Wall Motion
}

\author{
Natalie F. A. Edwards, MCardiac Ultrasound, BExSci, ACS, AMS, FASE, AFASA, \\ Gregory M. Scalia, MBBS, MMedSc, FRACP, FACC, FCSANZ, FASE, Kenji Shiino, MD, PhD, \\ Surendran Sabapathy, DPhil, BExScHons, \\ Bonita Anderson, DMU (Cardiac), MAppSc(MedUlt), ACS, AMS, FASE, FASA, \\ Robert Chamberlain, BSc(Hons), GradDipCardiacUlt, Bijoy K. Khandheria, MD, FASE, FACC, \\ and Jonathan Chan, MBBS, PhD, FRACP, FRCP, FSCCT, FACC, Brisbane and Gold Coast, Australia; and \\ Milwankee, Wisconsin
}

Background: Noninvasive detection of functionally significant coronary artery disease (CAD) by echocardiography remains challenging, with the need to perform stress imaging to detect ischemia. The aim of this study was to determine whether global myocardial work (MW), derived from noninvasive left ventricular (LV) pressure-strain loops at rest, can predict significant CAD in patients without regional wall motion abnormalities and preserved LV ejection fraction (EF).

Methods: One hundred and fifteen patients referred for coronary angiography who had $E F \geq 55 \%$, no resting regional wall motion abnormalities, and no chest pain were assessed using echocardiography. Global MW was derived from noninvasive LV pressure-strain loops constructed from speckle-tracking echocardiography indexed to brachial systolic blood pressure. Global constructive work represented the sum of positive work due to myocardial shortening during systole and negative work due to lengthening during isovolumic relaxation. Global wasted work represented energy loss by myocardial lengthening in systole and shortening in isovolumic relaxation. Global MW efficiency was derived from the percentage ratio of constructive work to the sum of constructive work and wasted work.

Results: Patients with significant CAD demonstrated a significantly reduced global MW $(P<.001)$ compared with those without CAD. Global longitudinal strain was significantly reduced $(P<.001)$ in patients with multivessel CAD but not those with single-vessel CAD $(P=.47)$. Receiver operating characteristic curve analysis demonstrated that global MW was the most powerful predictor of significant CAD (area under the curve $=0.786$ ) and was superior to global longitudinal strain (area under the curve $=0.693$ ). The optimal cutoff global MW value to predict significant CAD was $1,810 \mathrm{~mm} \mathrm{Hg} \%$ (sensitivity, $92 \%$; specificity, $51 \%$ ).

Conclusions: Noninvasive global MW derived using LV pressure-strain loops at rest is a more sensitive index than global longitudinal strain to detect significant $C A D$ in patients with no regional wall motion abnormalities and normal EF. This is a potential valuable clinical tool to assist in the early diagnosis of CAD. (J Am Soc Echocardiogr 2019; $\mathbf{\square}: \mathbf{\square}-\boldsymbol{\square}$.)

Keywords: Myocardial work, Strain, Speckle-tracking, Coronary artery disease

From the Department of Cardiology, The Prince Charles Hospital (N.F.A.E., G.M.S., B.A., R.C., J.C.); the School of Medicine, University of Queensland (G.M.S.), Brisbane; the School of Medicine and Menzies Health Institute Queensland, Griffith University, Gold Coast (N.F.A.E., K.S., S.S., J.C.), Australia; and the Aurora Cardiovascular and Thoracic Service Line, Aurora Healthcare, University of Wisconsin School of Medicine and Public Health, Milwaukee, Wisconsin (B.K.K.).

This research was supported by the CATHARSIS research grant from The Prince Charles Hospital Foundation and the Common Good (grant ER2016-014).

Conflicts of Interest: None.
Reprint requests: Jonathan Chan, MBBS, PhD, FRACP, FRCP, FSCCT, FACC, The Prince Charles Hospital, Department of Cardiology, Rode Road, Chermside, QLD 4032, Australia (E-mail: jonathan.chan@griffith.edu.au).

0894-7317/\$36.00

Crown Copyright 2019. Published by Elsevier Inc. on behalf of the American Society of Echocardiography. All rights reserved.

https://doi.org/10.1016/j.echo.2019.02.014 


\begin{tabular}{|l|}
\hline \multicolumn{1}{|c|}{ Abbreviations } \\
\hline 2D = Two-dimensional \\
AUC = Area under the \\
receiver operating \\
characteristic curve \\
CAD = Coronary artery \\
disease \\
CW = Constructive work \\
EF = Ejection fraction \\
GLS = Global longitudinal \\
strain \\
GWE = Global myocardial \\
work efficiency \\
ICC = Intraclass correlation \\
coefficient \\
LV = Left ventricular \\
MW = Myocardial work \\
RWMA = Regional wall \\
motion abnormality \\
STE = Speckle-tracking \\
echocardiography \\
TTE = Transthoracic \\
echocardiography \\
WW = Wasted work
\end{tabular}

Early detection and intervention is paramount in patients with significant coronary artery disease (CAD) to prevent adverse cardiac events, including myocardial infarction leading to left ventricular (LV) dysfunction. During the early stages of the disease process, global LV functional parameters of volume and ejection fraction (EF) are usually preserved. ${ }^{1-3}$ Unless there has been a previous infarction or stunning of the myocardium, regional wall motion abnormalities (RWMAs) are not evident at rest, and noninvasive detection of ischemia by transthoracic echocardiography (TTE) requires stress provocation. Stress echocardiography remains a widely accepted imaging modality for the assessment of CAD, but it is limited by operator dependence, subjectivity, and qualitative interpretation of RWMAs as well as a failure to reach a diagnostic target heart rate. $^{4,5}$

Myocardial deformation imaging using two-dimensional (2D) speckle-tracking echocardiography (STE) is a valuable tool, providing comprehensive quantitative assessment of myocardial function beyond EF and qualitative assessment of RWMAs. ${ }^{6-11}$ Previous studies have shown that peak systolic longitudinal strain is reduced in patients with significant $C A D$, even when resting LV wall motion and EF are preserved. ${ }^{2}$ However, one of the main limitations of STE is load dependency, which can affect the diagnostic accuracy of myocardial function evaluation. ${ }^{11,12}$ An increase in afterload has been shown to decrease strain, leading to misinterpretation of the true contractile function of the myocardium. ${ }^{12-18}$

Myocardial work (MW) accounts for deformation as well as afterload through interpretation of strain in relation to dynamic noninvasive LV systolic pressure. ${ }^{18-22}$ Early work looking at MW showed that patients with systolic blood pressure $\geq 160 \mathrm{~mm} \mathrm{Hg}$ displayed significantly higher global MW compared with that seen in control subjects, despite strain and EF being preserved. ${ }^{22}$ This confirmed that conventional echocardiographic parameters are limited in detecting the increased work and load imposed on the LV myocardium. ${ }^{22}$

MW may identify early abnormalities in LV function and may establish a more sensitive index for early stage LV dysfunction. The aim of this study was to explore the incremental value of MW in the detection of significant CAD at rest in patients with normal LV function and without RWMAs.

\section{METHODS}

\section{Study Cohort}

This was a single-center cross-sectional study prospectively recruiting patients referred for clinically indicated coronary angiography.
The clinical indications for coronary angiography were history of chest pain $(n=107)$, history of exertional dyspnea $(n=46)$, nonST-segment elevation myocardial infarction $(n=20)$, positive results on exercise stress echocardiography $(n=14)$, positive results on exercise stress testing $(n=13)$, positive results on myocardial perfusion scan $(n=25)$, positive results on computed tomographic coronary angiography $(n=14)$, and history of mild CAD $(n=3)$. None of the patients had chest pain at the time of the echocardiographic study. This study was approved by the human research and ethics commit tee of the research institution, and all patients provided informed consent. TTE was performed on patients $<3$ hours before cardiac catheterization. A total of 115 patients fulfilled the inclusion criteria of normal EF, defined as $\geq 55 \%$, without observed resting RWMAs. Patients were excluded if there was significant aortic stenosis, prosthetic aortic valve replacement, hypertrophic cardiomyopathy, other significant valvular disease, or paced rhythm. Because of variation in $\mathrm{R}-\mathrm{R}$ intervals, patients in atrial fibrillation were excluded. Patients with left bundle branch block and paced rhythm were excluded because of the possible presence of abnormal septal motion.

\section{Echocardiographic Analysis}

Comprehensive TTE was performed by experienced sonographers using a Vivid E95 ultrasound system equipped with an M5S 3.5$\mathrm{MHz}$ transducer (GE Vingmed Ultrasound, Horten, Norway) $<3$ hours before coronary angiography. Patients were scanned in the left lateral decubitus position for optimal image quality. Standard 2D images, consisting of three cardiac cycles triggered to the QRS complex, were saved in cine-loop digital format for offline analysis. LVend-diastolic and end-systolic volumes were calculated using the modified biplane Simpson method, with EF subsequently determined. LV mass was obtained using a linear 2D approach, with LV mass index calculated as the anatomic mass divided by body surface area. All 2D and Doppler recordings and measurements were performed according to American Society of Echocardiography guidelines. $^{23,24}$

\section{Two-Dimensional STE}

Two-dimensional grayscale images from the apical four-chamber, two-chamber, and long-axis views were acquired at frame rates between 50 and 80 frames $/ \mathrm{sec}$ (mean, $56.8 \pm 2.8$ frames $/ \mathrm{sec}$ ) to enable global longitudinal strain (GLS) analysis by STE. GLS was quantified by an observer blinded to coronary angiographic results using semiautomated function imaging from vendor-specific offline analysis software (EchoPAC version 202; GE Vingmed Ultrasound). Following the identification of aortic valve closure from the apical long-axis view using the automated function, three index points were used to define the mitral annulus and LV apex at the end-systolic frame in each apical view. The automated algorithm traces and tracks the LV myocardium, with adjustments made as necessary. Using the 17segment model, the software calculated GLS from the weighted average of the peak systolic longitudinal strain of all segments. For the purposes of this study, GLS is expressed as absolute values. Patients were excluded from the study if regional tracking of one or more segments was suboptimal.

\section{MW Analysis}

The methodology of MW calculation from noninvasive LV pressure-strain analysis along with its validation has been described previously. ${ }^{21,25} \mathrm{MW}$ and related indices were calculated using the 


\section{HIGHLIGHTS}

- Calculation of myocardial work incorporates both strain and afterload.

- Myocardial work can now be derived from noninvasive pressure-strain loops.

- Myocardial work is superior to GLS in predicting CAD.

- Myocardial work can assess early subclinical ischemic myocardial dysfunction.

latest vendor-specific module within the Automated Function Imaging software (EchoPAC Version 202) using a combination of LV strain data and a noninvasively estimated LV pressure curve. Blood pressure was measured by sphygmomanometry immediately before TTE, with peak systolic LV pressure assumed to be equal to peak arterial pressure. The MW software then constructed a noninvasive LV pressure curve adjusted according to the duration of isovolumic and ejection phases defined by the timing of aortic and mitral valve opening and closing events on $2 \mathrm{D}$ echocardiography. ${ }^{12,16} \mathrm{LV}$ strain and pressure data were then synchronized through alignment of valvular timing events and systolic blood pressure.

Global MW was quantified by calculating the rate of regional shortening by differentiation of the strain tracing and multiplying by instantaneous LV pressure. This instantaneous measure of power was this integrated over time to measure MW as a function of time during systole (time interval from mitral valve closure through to mitral valve opening). During LV ejection, segments were analyzed for wasted work (WW) and/or constructive work (CW; Figure 1A), with global values determined as the averages of all segmental values and displayed on the LV pressure-strain loop diagram (Figures $1 \mathrm{~B}$ and $1 \mathrm{C}$ ). The following parameters were generated by the software:

1. Global MW (mm Hg \%), area within the global LV pressure-strain loop

2. Global myocardial $\mathrm{CW}$ ( $\mathrm{mm} \mathrm{Hg} \mathrm{\% ),} \mathrm{an} \mathrm{estimate} \mathrm{of} \mathrm{the} \mathrm{work} \mathrm{per-}$ formed by the LV segments consisting of shortening during systole plus lengthening in isovolumic relaxation

3. Global myocardial WW (mm Hg \%), an estimate of negative work of the LV segments consisting of myocardial lengthening during systole plus any shortening during isovolumic relaxation

4. Global MW efficiency (GWE; \%), constructive MW divided by the sum of CW and WW, expressed as a percentage (these values are not affected by peak LV pressure)

An example of these parameters is presented in Figure 1. Global LV MW is represented in blue, work performed by the mid inferoseptal segment is represented in orange, and the mid anterolateral segment is represented in gray. The area under the global MW curve (blue) from mitral valve closure to mitral valve opening is the global MW index value. The area under each curve is also reflected as the area within the LV pressure-strain loop diagrams (Figure 1B for the mid inferoseptal segment, Figure $1 \mathrm{C}$ for the mid anterolateral segment).

When work falls below the zero baseline (as seen in the mid anterolateral segment), it reflects segmental elongation (when the LV myocardium should be shortening during systole) and is equivalent to WW (Figure 1A). In comparison, MW is positive during systole in the mid inferoseptum and is equivalent to CW. WW can also be visually appreciated in the mid anterolateral segment (Figure 1C, represented in green) on the regional LV pressure-strain loop as the segment elongates during LV contraction. The value for global MW can be obtained from the pressure-strain loop diagram (Figures 1B, $1 \mathrm{C}$, and 2), whereas CW and WW parameters are derived from the contraction pattern of regional segments.

\section{Coronary Angiography}

All patients underwent clinically indicated coronary angiography within 3 hours of TTE. All coronary angiographic images were evaluated by experienced operators blinded to the echocardiographic results. Significant CAD was defined as $\geq 70 \%$ luminal narrowing in one or more major epicardial vessels by visual assessment. $^{24}$

\section{Statistical Analysis}

All statistical analyses were performed using SPSS version 25.0 (SPSS, Chicago, IL). Continuous variables are expressed as mean \pm SD. Categorical variables are presented as numbers and percentages. Normal distribution of the data were verified using the Shapiro-Wilk test. Comparison of continuous variable within only two groups was performed using an independent $t$ test, with Levene's test performed before the test for equality of variances. Comparison of continuous variables among more than two groups was performed using a one-way analysis of variance, with Tukey post hoc adjustments performed when significant differences were detected. Nonparametric analysis of overall values of sensitivity and specificity as well as area under the receiver-operating characteristic curve (AUC) were applied. Receiver operating characteristic curve analysis was used to assess optimal cutoff points for MW and GLS in the detection of significant CAD. Categorical variables were compared using the $\chi^{2}$ test, as indicated. All tests were two-sided, and $P$ values $<.05$ were considered to indicate statistical significance.

\section{Intra- and Interobserver Variability}

Twenty-five patients were randomly selected, and MW parameters were measured by two observers blinded to patients' clinical data and each other's results. Intraobserver variability was assessed using offline data at different points in time. Interobserver variability was determined by repeating measurements from the same images. Intraand interobserver variability was calculated using intraclass correlation coefficients (ICCs) and SEM.

\section{RESULTS}

\section{Patient Characteristics}

One hundred fifteen patients satisfied the baseline inclusion criteria. Six patients were excluded from strain and MW analysis because of inadequate image quality, with suboptimal regional tracking of any segment in a single view (feasibility, 95\%). None of the patients described chest pain, and there was no evidence of electrocardiographic evidence of ischemia during the echocardiographic study. A total of 109 patients (mean age, $66.7 \pm 11$ years; 71 men) were therefore evaluated in the study and were initially divided into two groups, no CAD $(n=28)$ and significant CAD ( $\geq 70 \%$ stenosis; $n=81)$, on the basis of the results of coronary angiography performed within 3 hours following TTE. Patients with significant CAD were then further subdivided into those with single-vessel CAD $(n=31)$ and 

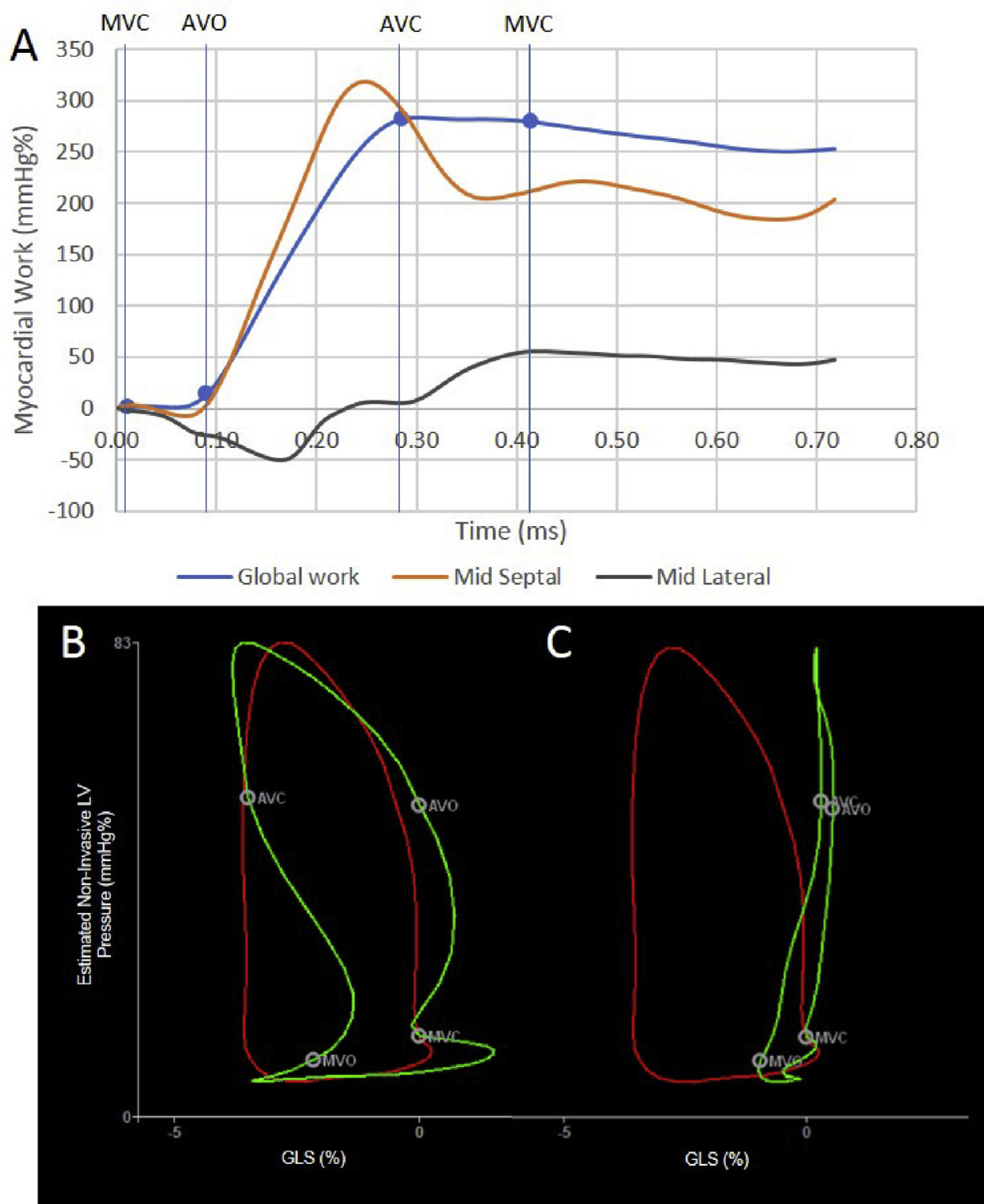

Figure 1 (A) Global LV MW is represented in blue, work performed by the mid inferoseptal segment is represented in orange, and work performed by the mid anterolateral segment is represented in gray. (B) LV pressure-strain loop diagram. Global MW is represented in red, and regional work performed by the mid inferoseptal segment is represented in green. (C) LV pressure-strain loop diagram. Global MW is represented in red, and regional work performed by the mid anterlateral segment is represented in green. AVC, Aortic valve closure; AVO, aortic valve opening; $M V C$, mitral valve closure; $M V O$, mitral valve opening.

those with multivessel CAD $(n=50)$. Medications such as $\beta$-blockers, angiotensin-converting enzyme inhibitors, angiotensin II receptor blockers, statins, and nitrates were more common among patients with significant CAD.

Patient demographic data according to the study groups are presented in Table 1. Patients with CAD were, as a group, significantly older than control subjects (mean difference, $-6.9 \pm 2.4$ years; $P<.001)$, with the multivessel CAD $(P<.05)$ group the primary contributors to this age difference. There was no significant difference in systolic blood pressure between patients with no CAD and those with CAD. According to a $\chi^{2}$ test, there were no significant differences in cardiac rhythm $(P=.68)$, or coronary dominance $(P=.95)$, across groups.

\section{No CAD Compared with Significant CAD}

Conventional echocardiographic data are presented in Table 2. There were no significant differences in 2D biplane volumes or EF between patients without CAD and those with CAD. LV mass index was significantly higher $(P<.05)$ in patients with CAD. The average $\mathrm{e}^{\prime}$ was significantly lower $(P<.05)$ and average $\mathrm{E} / \mathrm{e}^{\prime}$ ratio significantly higher $(P<.05)$ in patients with CAD, but no correlations were seen be tween $\mathrm{MW}$ and $\mathrm{E} / \mathrm{e}^{\prime}$ ratio. 


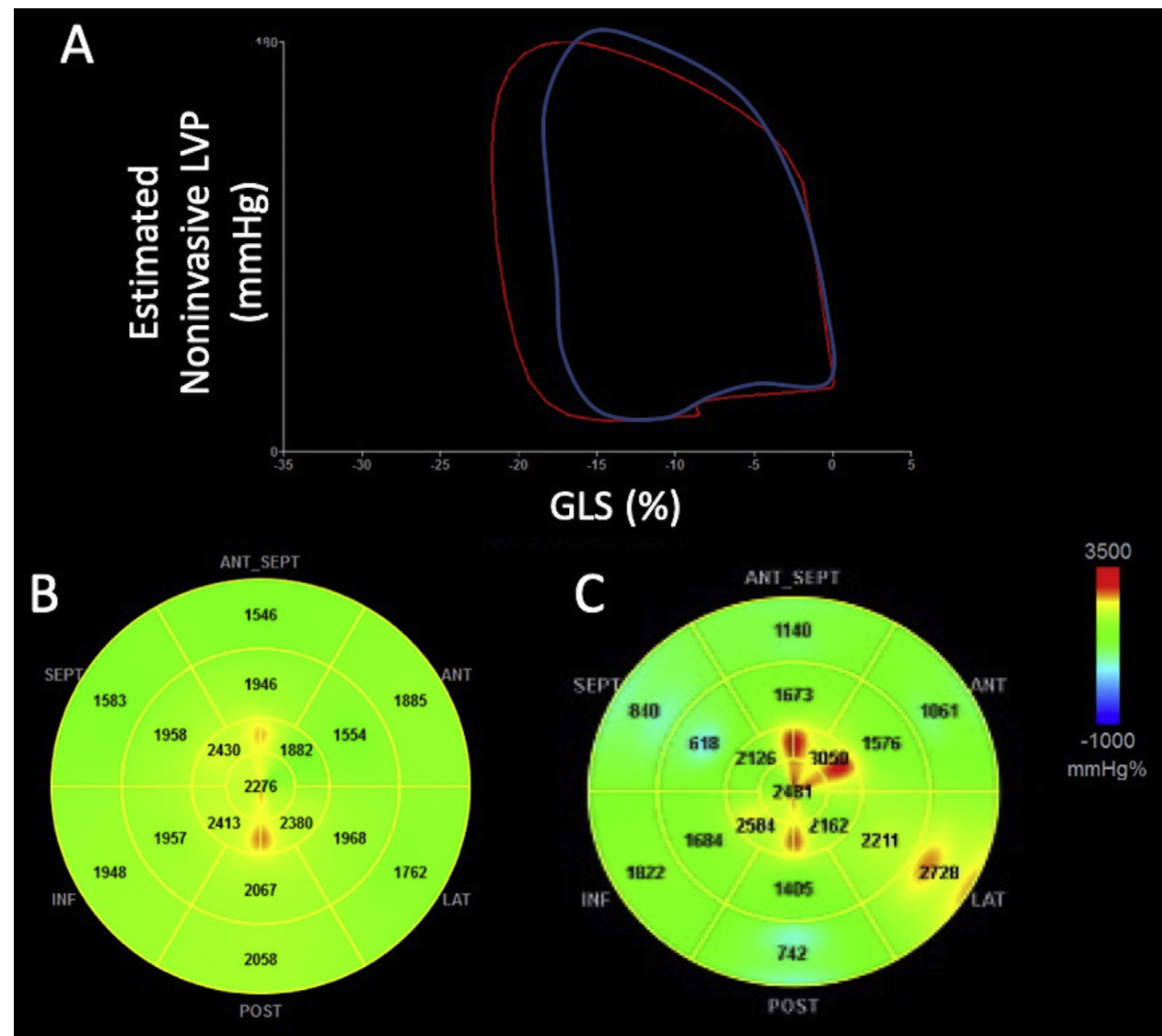

Figure 2 (A) Noninvasive LV pressure-strain loop diagram. The LV pressure-strain loop follows a counterclockwise direction. Following mitral valve closure (MVC), there is an initial rapid rise in LV pressure but minimal change in strain (0\%) during the period of isovolumic contraction (IVC). After aortic valve opening (AVO), LV ejection leads to an increase in GLS (becoming more negative) with myocardial deformation reaching its highest point just as LV pressure begins to decline. LV pressure then falls rapidly with little change in GLS (isovolumic relaxation [IVR]). Once LV pressure falls below left atrial pressure in early diastole and after mitral valve opening (MVO), the left ventricle rapidly relaxes during diastasis. The area within the loop is estimated as global MW. The red loop represents a patient with no angiographic evidence of CAD. The blue loop represents a patient with multivessel CAD. Visually the LV pressure-strain loop has a smaller area because GLS is reduced (similar systolic blood pressure [SBP] between patients), which is confirmed with the significantly reduced MW parameters. (B) Segmental bull's-eye MW plot from a patient with no angiographic evidence of CAD (GLS 18\%, global MW 2,016 mm Hg \%, global constructive MW 2,460 mm Hg \%, GWE 98\%, SBP $146 \mathrm{~mm} \mathrm{Hg}$ ). (C) Segmental bull's-eye MW plot from a patient with multivessel CAD (GLS 16\%, global MW 1,857 mm Hg \%, global constructive MW 2,073 mm Hg \%, GWE 97\%, SBP $145 \mathrm{~mm} \mathrm{Hg}$ ). On the bull's-eye plot, there is significant reduction in regional MW demonstrated as blue-coded areas. ANT, Anterior; ANT_SEPT, anterior septal; INF, inferior; LAT, lateral; POST, posterior; SEPT, septal.

LV GLS $(P<.05)$ and global MW $(P<.001)$ were significantly lower in all patients with CAD compared with those without CAD, despite no RWMAs and preserved EFs (Table 3 ). Reduced MW is also evident on the noninvasive LV pressure-strain loop diagram (Figure 2A). Compared with a patient with no angiographically significant CAD (shown in red), the LV pressure-strain loop of a patient with multivessel CAD showed a smaller loop and area (shown in blue). The 17-segment bull's-eye plot showed a uniform MW distribution (coded in green) through the myocardial segments for the patient with no CAD (Figure 2B), while there are regions of reduced MW (coded in blue) in the patient with multivessel CAD (Figure 2C).

Global CW was significantly reduced $(P<.001)$ in patients with CAD compared with those without CAD, with no significant difference in global WW $(P=.41)$. Because GWE is derived by dividing $\mathrm{CW}$ by the sum of $\mathrm{CW}$ and $\mathrm{WW}$, this parameter was also significantly reduced $(P<.05)$ in CAD.

Receiver operating characteristic curve analysis was used to discriminate whether MW parameters and GLS were able to predict
CAD (Figure 3). Global MW was superior to GLS (Figure 3A), with an AUC of $0.786(P<.001$; Figure 3B, Table 4$)$. Other predictors of $\mathrm{CAD}$ were global $\mathrm{CW}$ (AUC $=0.746$; Figure $3 \mathrm{C}$ ) and $\mathrm{GWE}$ (AUC $=0.650$; Figure 3D). According to the receiver operating characteristic curve analysis, the optimal cutoff value for global MW for the detection of patients diagnosed with CAD was $1,810 \mathrm{~mm}$ $\mathrm{Hg} \%$, with sensitivity of $92.0 \%$, specificity of $51.0 \%$, positive predictive value of $95.0 \%$, and negative predictive value of $40.5 \%$. The optimal cutoff value for GLS was $16.7 \%$, with sensitivity of $89.3 \%$ and specificity of $61.8 \%$.

\section{No CAD Compared with Single- and Multivessel CAD}

MW parameters were significantly reduced $(P<.05)$ in both singleand multivessel CAD groups (Table 5). However, GLS was reduced only in multivessel CAD and not significantly reduced $(P=.47)$ in those with single-vessel CAD. GWE was not reduced enough in CAD subgroups to be significant. 
Table 1 Clinical characteristics of patients in the study groups by angiographic evaluation

\begin{tabular}{|c|c|c|c|c|}
\hline Variable & No CAD $(n=28)$ & All significant CAD $(n=81)$ & Single-vessel CAD $(n=31)$ & Multivessel CAD $(n=50)$ \\
\hline Age $(y)$ & $61 \pm 13$ & $68 \pm 10^{*}$ & $66 \pm 10$ & $69 \pm 10^{\star}$ \\
\hline Male & $12(43)$ & $59(73)^{\star}$ & $19(61)^{\star}$ & $41(82)^{\star}$ \\
\hline $\mathrm{BSA}\left(\mathrm{m}^{2}\right)$ & $2.1 \pm 0.3$ & $2.0 \pm 0.2$ & $2.0 \pm 0.2$ & $2.0 \pm 0.2$ \\
\hline Heart rate (beats/min) & $65.6 \pm 11.8$ & $59.7 \pm 8.7$ & $60.0 \pm 10.0$ & $60.5 \pm 9.6$ \\
\hline Systolic BP (mm Hg) & $137 \pm 13$ & $136 \pm 20$ & $130 \pm 18$ & $139 \pm 20$ \\
\hline Diastolic BP (mm Hg) & $79 \pm 8$ & $74 \pm 10$ & $74 \pm 10$ & $75 \pm 9$ \\
\hline \multicolumn{5}{|l|}{ Cardiac rhythm } \\
\hline Sinus rhythm & $26(93)$ & $70(86)$ & $28(90)$ & $42(84)$ \\
\hline Sinus with LBBB & $2(7)$ & $9(11)$ & $3(10)$ & $6(12)$ \\
\hline First-degree AV block & $0(0)$ & $2(2)$ & $0(0)$ & $2(4)$ \\
\hline \multicolumn{5}{|l|}{ Coronary dominance } \\
\hline Right coronary dominance & $23(81)$ & $71(88)$ & $26(84)$ & $45(90)$ \\
\hline Left coronary dominance & $2(7)$ & $5(6)$ & $2(6)$ & $3(6)$ \\
\hline Codominance & $3(11)$ & $5(6)$ & $3(10)$ & $2(4)$ \\
\hline \multicolumn{5}{|l|}{ Comorbidities } \\
\hline Hypertension & $15(54)$ & $59(72)^{*}$ & $26(84)^{\star}$ & $33(66)$ \\
\hline Diabetes mellitus & $4(14)$ & $25(31)^{*}$ & $6(19)$ & $19(38)^{*}$ \\
\hline Hypercholesterolemia & $13(46)$ & $44(54)$ & $19(61)^{\star}$ & $25(50)$ \\
\hline Family history & $7(25)$ & $29(36)$ & $11(35)$ & $18(36)$ \\
\hline \multicolumn{5}{|l|}{ Medications } \\
\hline$\beta$-blockers & $2(7)$ & $27(33)^{\star}$ & $7(22)^{*}$ & $20(40)^{*}$ \\
\hline Calcium channel blockers & $1(4)$ & $17(21)^{\star}$ & $6(19)^{*}$ & $11(22)^{\star}$ \\
\hline ACE inhibitors & $3(11)$ & $14(17)$ & $6(19)$ & $8(16)^{*}$ \\
\hline ARBs & $1(4)$ & $14(17)^{\star}$ & $3(10)$ & $11(22)^{*}$ \\
\hline Statins & $3(11)$ & $40(49)^{\star}$ & $13(42)^{\star}$ & $27(54)^{\star}$ \\
\hline Nitrates & $0(0)$ & $30(37)^{\star}$ & $11(35)^{\star}$ & $19(38)^{\star}$ \\
\hline
\end{tabular}

$A C E$, Angiotensin-converting-enzyme; $A R B$, angiotensin II receptor blocker; $A V$, atrioventricular; $B P$, blood pressure; $B S A$, body surface area; $\angle B B B$, left bundle branch block.

Data are expressed as mean \pm SD or as number (percentage).

*Significantly different $(P<.05)$ compared with the control group.

Table 2 Conventional echocardiographic parameters in control subjects compared with all patients with significant CAD

\begin{tabular}{lcccc}
\hline \multicolumn{1}{c}{ Variable } & No CAD $(n=28)$ & All significant CAD $(n=81)$ & Single-vessel CAD $(n=31)$ & Multivessel CAD $(n=50)$ \\
\hline LVDd $(\mathrm{mm})$ & $47 \pm 5$ & $48 \pm 4$ & $47 \pm 5$ & $33 \pm 4$ \\
LVDs $(\mathrm{mm})$ & $32 \pm 4$ & $32 \pm 4$ & $1.1 \pm 0.2$ & $32 \pm 4$ \\
IVSd (cm) & $1.0 \pm 0.1$ & $1.1 \pm 0.1^{*}$ & $1.0 \pm 0.2$ & $1.1 \pm 0.1^{*}$ \\
PWd (cm) & $1.0 \pm 0.1$ & $1.0 \pm 0.1$ & $182 \pm 47$ & $1.1 \pm 0.1^{*}$ \\
LV mass $(\mathrm{g})$ & $170 \pm 43$ & $187 \pm 42$ & $93 \pm 23$ & $193 \pm 41$ \\
LV mass index $\left(\mathrm{g} / \mathrm{m}^{2}\right)$ & $85 \pm 17$ & $95 \pm 20^{*}$ & $107 \pm 33$ & $98 \pm 20^{*}$ \\
Biplane EDV $(\mathrm{mL})$ & $103 \pm 30$ & $108 \pm 33$ & $40 \pm 14$ & $63 \pm 4$ \\
Biplane ESV $(\mathrm{mL})$ & $39 \pm 13$ & $42 \pm 15$ & $63 \pm 109$ \\
Biplane EF $(\%)$ & $62 \pm 5$ & $61 \pm 4$ & $60 \pm 4$ \\
\hline
\end{tabular}

$E D V$, End-diastolic volume; ESV, end-systolic volume; IVSd, interventricular septal thickness in diastole; LVDd, LV dimension in diastole; LVDs, LV dimension in systole; $P W d$, posterior wall thickness in diastole.

Data are expressed as mean $\pm \mathrm{SD}$.

${ }^{\star}$ Significantly different $(P<.05)$ compared with the control group. 
Table 3 GLS and MW parameters in control subjects compared with all patients with significant CAD

\begin{tabular}{|c|c|c|c|c|c|c|c|}
\hline \multirow[b]{2}{*}{ Variable } & \multirow[b]{2}{*}{ No CAD $(n=28)$} & \multirow[b]{2}{*}{ All significant CAD ( $n=81)$} & \multirow[b]{2}{*}{$P$} & \multirow[b]{2}{*}{ Mean difference } & \multirow[b]{2}{*}{ SE } & \multicolumn{2}{|c|}{$95 \% \mathrm{Cl}$} \\
\hline & & & & & & Lower & Upper \\
\hline GLS (\%) & $19.0 \pm 2.4$ & $17.2 \pm 2.4^{*}$ & .002 & 1.73 & 0.53 & 0.65 & 2.81 \\
\hline Global MW (mm Hg \%) & $2,158 \pm 299$ & $1,801 \pm 352^{*}$ & .001 & 356.3 & 69.4 & 217.4 & 495.3 \\
\hline GWE (\%) & $96 \pm 2$ & $94 \pm 3^{*}$ & .016 & 1.449 & 0.594 & 0.271 & 2.628 \\
\hline Global CW (mm Hg \%) & $2,494 \pm 430$ & $2,146 \pm 430^{*}$ & .001 & 347.4 & 80.7 & 186.1 & 508.7 \\
\hline Global WW (mm Hg \%) & $93 \pm 53$ & $103 \pm 58$ & .411 & -9.95 & 12.0 & -34.0 & 14.1 \\
\hline
\end{tabular}

*Significantly different $(P<.05)$ compared with the control group.
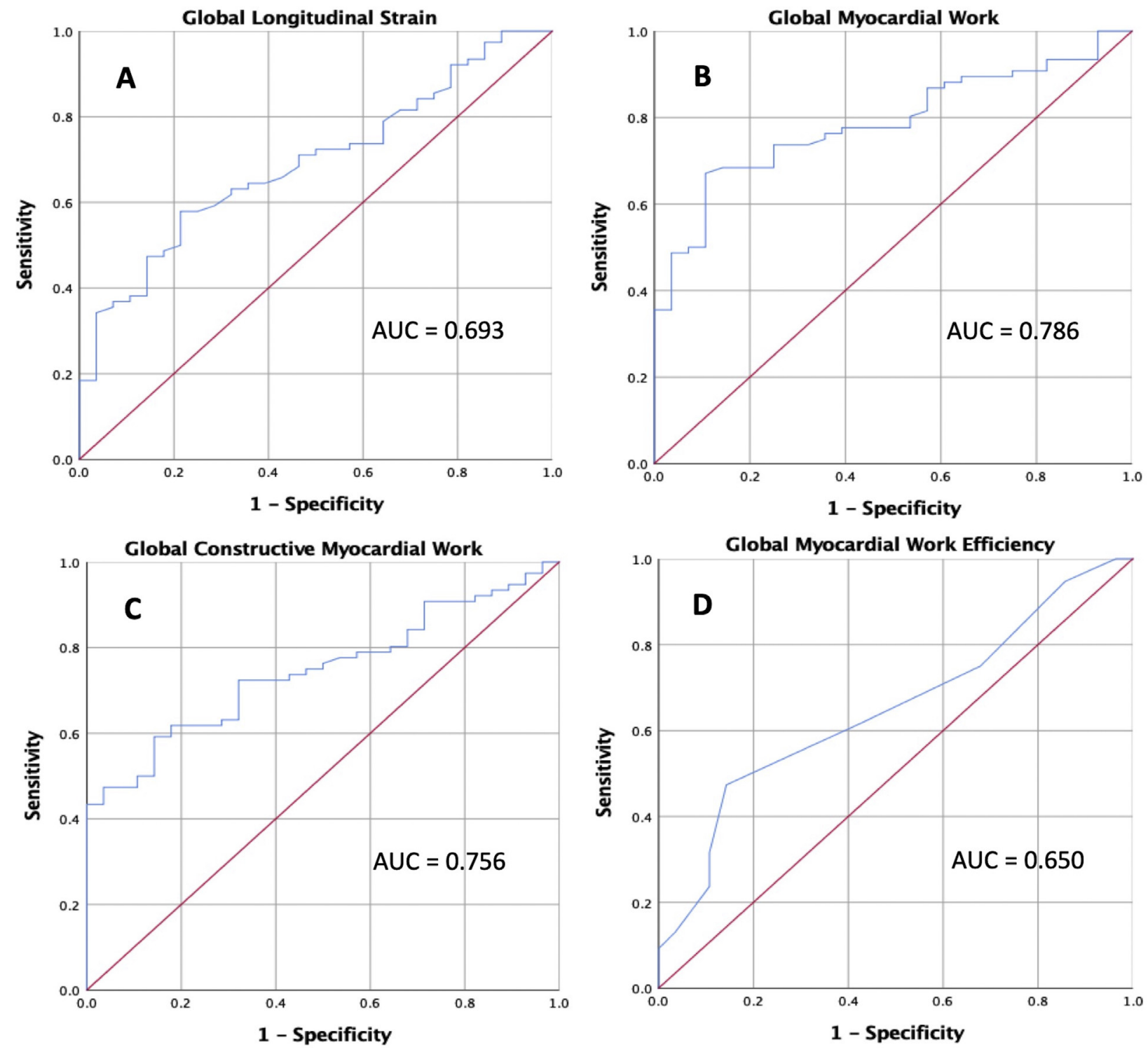

Figure 3 Receiver operating characteristic curve for the detection of CAD. (A) GLS. (B) Global MW. (C) Global CW. (D) Global MW efficiency. Global MW was the most powerful predictor of CAD (AUC =0.786; 95\% Cl, 0.699-0.874; $P<.001)$. A valve of $1,998 \mathrm{~mm} \mathrm{Hg}$ $\%$ had the most optimal sensitivity of $78 \%$ and specificity of $61 \%$. Global CW was also a strong predictor of CAD (AUC $=0.746 ; 95 \%$ $\mathrm{Cl}, 0.653-0.839 ; P<.001)$. GLS was less predictive in this study cohort (AUC = 0.693; 95\% Cl, 0.588-0.798; $P<.05$ ). 
Table 4 Receiver operating characteristic curve analysis for the detection of CAD

\begin{tabular}{lccccc}
\hline & GLS (\%) & Global MW (mm Hg \%) & GWE (\%) & Global CW (mm Hg \%) & Global WW (mm Hg \%) \\
\hline AUC (SE) & $0.693(0.054)^{\star}$ & $0.786(0.045)^{\star}$ & $0.650(0.057)^{*}$ & $0.746(0.047)^{\star}$ & $0.458(0.062)$ \\
AUC 95\% Cl & $0.588-0.798$ & $0.699-0.874$ & $0.537-0.762$ & $0.653-0.839$ & $0.337-0.579$ \\
Cutoff value & $16.7 \%$ & 1,810 & $93.5 \%$ & 2,071 & 80 \\
Sensitivity & $89.3 \%$ & $92.0 \%$ & $89.3 \%$ & $66.4 \%$ & $56.6 \%$ \\
Specificity & $61.8 \%$ & $51.0 \%$ & $68.4 \%$ & $60.5 \%$ \\
\hline
\end{tabular}

*Significantly different $(P<.05)$ compared with the control group.

Table 5 GLS and MW parameters in patients with all significant, single-vessel, and multivessel CAD

\begin{tabular}{|c|c|c|c|c|}
\hline Variable & No CAD $(n=28)$ & All significant CAD $(n=81)$ & Single-vessel CAD $(n=31)$ & Multivessel CAD $(n=50)$ \\
\hline GLS (\%) & $19.0 \pm 2.4$ & $17.3 \pm 2.4^{*}$ & $18.0 \pm 2.3$ & $16.9 \pm 2.7^{\star}$ \\
\hline Global MW (mm Hg \%) & $2,158 \pm 299$ & $1,816 \pm 352^{*}$ & $1,810 \pm 351^{*}$ & $1,823 \pm 365^{\star}$ \\
\hline GWE (\%) & $95.6 \pm 2.0$ & $94.3 \pm 2.9$ & $94.6 \pm 3.0$ & $94.1 \pm 2.8$ \\
\hline Global CW (mm Hg \%) & $2494 \pm 337$ & $2156 \pm 427^{\star}$ & $2151 \pm 432^{*}$ & $2152 \pm 427^{\star}$ \\
\hline Global WW (mm Hg \%) & $93 \pm 53$ & $101 \pm 57$ & $97 \pm 57$ & $103 \pm 57$ \\
\hline
\end{tabular}

*Significantly different $(P<.05)$ compared with the control group.

Table 6 ICCs for intra- and interobserver variability for MW parameters and GLS

\begin{tabular}{|c|c|c|c|c|c|c|}
\hline \multirow[b]{2}{*}{ Variable } & \multicolumn{3}{|c|}{ Interobserver variability } & \multicolumn{3}{|c|}{ Intraobserver variability } \\
\hline & ICC & $95 \% \mathrm{Cl}$ & SEM & ICC & $95 \% \mathrm{Cl}$ & SEM \\
\hline Global MW (mm Hg \%) & 0.953 & $0.863-0.981$ & 215.9 & 0.973 & $0.939-0.988$ & 148.5 \\
\hline GWE (\%) & 0.988 & $0.973-0.995$ & 1.4 & 0.991 & $0.980-0.996$ & 1.3 \\
\hline Global CW (mm Hg \%) & 0.967 & $0.889-0.987$ & 212.0 & 0.972 & $0.937-0.988$ & 179.2 \\
\hline Global WW (mm Hg \%) & 0.941 & $0.867-0.974$ & 32.8 & 0.965 & $0.921-0.984$ & 25.1 \\
\hline GLS (\%) & 0.949 & $0.793-0.982$ & 1.5 & 0.976 & $0.945-0.989$ & 0.93 \\
\hline
\end{tabular}

\section{Intra- and Interobserver Variability}

All MW parameters and GLS exhibited excellent intra- and interobserver correlation, with ICC values $>0.94$ (Table 6). ICC values were higher for interobserver variability when the same images were analyzed by the two different observers. In comparison, ICC was only slightly lower, but with excellent correlation for intraobserver variability when the same observer performed offline measurements on different images obtained at the time of the echocardiographic study.

\section{DISCUSSION}

This study demonstrates that noninvasive MW provides quantifiable information for identification of patients with single- and multivessel CAD at rest despite the absence of visually detectable RWMAs. In summary, in patients with CAD, global MW and global CW are both reduced. Global WW is increased, and therefore myocardial efficiency is reduced. Prediction of CAD in patients with reduced global MW was superior to all other echocardiographic parameters, including GLS and EF. Furthermore, reproducibility of MW parameters was excellent. These findings suggest that MW is sensitive to metabolic adaptation of the myocardium in the presence of CAD and may also have clinical diagnostic utility in the early stages of the disease. In our study, we were able to define an optimal global MW cutoff value of $1,810 \mathrm{~mm} \mathrm{Hg} \%$ (sensitivity, 92\%; specificity, 51\%).

Noninvasive detection of patients with significant CAD remains a challenge despite the widespread use of imaging and exercise provocation to detect hemodynamic significance. Many patients referred for coronary angiography show normal results or nonobstructive CAD. ${ }^{26-28}$ Repetitive and intermittent ischemic insults on the LV myocardium may result in subtle forms of stunning that may reduce systolic longitudinal function, despite no obvious resting RWMAs. Because subendocardial fibers are more susceptible to ischemia, ${ }^{4,29,30}$ many studies have shown that longitudinal strain more accurately detects early derangement of the myocardium caused by ischemia. ${ }^{2,13,31-33}$ Because EF and visual assessment of regional wall motion are based on radial thickening of the endocardial border, these parameters are less sensitive to detect early ischemia. ${ }^{34,35}$ Therefore, longitudinal strain, which has a predominant contribution from the endocardial layer, more accurately detects early derangement of cardiac function caused by ischemia. ${ }^{2,13,31-33}$ In patients with normal EFs and absence of RWMAs, the discriminatory presence of reduced GLS provides 
incremental diagnostic value in the detection of underlying CAD. ${ }^{2,13,31-33}$ However, the optimal GLS diagnostic cutoff value varies significantly across the many studies, possibly because of clinical characteristics, afterload at the time of the acquisition, intervendor differences, and operator skills. 4,28,32,36-38

In this study cohort, GLS was only a fair predictor of CAD, with an AUC of 0.693 in patients with CAD and no chest pain at the time of the investigation. GLS was reduced in both single $(18.0 \pm 2.3 \%)$ and multivessel CAD $(16.9 \pm 2.7 \%)$, but it was only significantly reduced in patients with multivessel compared with those with no CAD $(19.0 \pm 2.4 \%)$. Significant multivessel disease exposes more of the LV myocardium to ischemia, and therefore it seems reasonable that GLS was lower in these patients with more of the longitudinally arranged subendocardial fibers affected by ischemia. Similarly, Choi et al. ${ }^{2}$ showed reduced GLS for all patients with CAD but only significantly reduced GLS in patients with high risk, three-vessel, and left main disease.

In comparison, Shimoni et al. ${ }^{32}$ found a significant reduction in GLS $(A U C=0.79)$ in hospitalized patients with chest pain, suggesting that a greater ischemic effect reduces GLS. In the assessment of patients with stable and unstable angina, Choi et $_{\text {al. }}^{2}$ found that resting GLS was significantly reduced in patients with severe CAD despite normal EFs and absence of RWMAs. In another recent study published by our research group, we were able to describe a change in LV pressure-strain pattern with significant reduction in MW in more advanced patients with established ischemic cardiomyopathy and reduced EFs. ${ }^{22}$

LV myocardial strain is a load-dependent variable influenced by alterations in loading conditions. ${ }^{12,14,15}$ A meta-analysis of 24 studies by Yingchoncharoen et al. ${ }^{13}$ found variations of normal ranges of strain associated with differences in systolic blood pressure, with an increase in afterload associated with reduction in LV strain. Skulstad et al. ${ }^{14}$ assessed the influence of loading conditions in an animal study and found that contractile patterns of ischemic myocardium are strongly influenced by loading conditions. The study found that following acute elevation in afterload, there was an immediate change from hypokinesis to dyskinesis. ${ }^{15}$ In contrast, noninvasive MW is less load dependent, accounting for the patient's systolic blood pressure at the time of performing STE.

Global MW was significantly reduced in all patient subgroups within this study, both single-vessel and multivessel CAD compared with patients with no CAD, despite no resting RWMAs or chest pain during echocardiography. Boe et al. ${ }^{18}$ investigated the use of $\mathrm{MW}$ in patients with non-ST-segment acute coronary syndromes and found regional $\mathrm{MW}<1,700 \mathrm{~mm} \mathrm{Hg} \%$ in more than four adjacent dysfunctional segments to be significantly superior to GLS or EF to detect CAD. Global MW was also significantly reduced in patients with acute coronary artery occlusion $(1,781 \pm 360 \mathrm{~mm} \mathrm{Hg} \%$, AUC $=0.81$ ). Interestingly, when afterload was increased, systolic blood pressure was a significant covariate, showing a further reduction in strain in the ischemia area. In the same study, strain measured in hypertensive patients with no evidence of acute coronary syndrome showed eight adjacent segments with impaired function. ${ }^{18}$ This may lead the interpreter to assuming there is myocardial dysfunction caused by CAD in these segments, when in fact they are affected by afterload. ${ }^{18}$ Because MW accounts for systolic blood pressure, these parameters showed there was normal work in these falsepositive hypertensive patients. ${ }^{21}$

MW assessed using noninvasive LV pressure-strain loops has been shown to reflect myocardial metabolic demand and oxygen consumption evaluated using ${ }^{18} \mathrm{~F}$-fluorodeoxyglucose positron emission tomography. ${ }^{21}$ Fluorine-18 fluorodeoxyglucose positron emission tomography provides information about perfusion as well as myocar- dial metabolism. ${ }^{15,39}$ When there is reduced blood flow and oxygen to regions of the myocardium, fatty acid metabolism shifts preferentially to glucose metabolism, with ${ }^{18} \mathrm{~F}$-fluorodeoxyglucose positron emission tomographic imaging showing regions of ischemia displayed as reduced levels of metabolism. ${ }^{15,39,40}$ The significant reductions in global MW and global CW in this study potentially reflect the pathologic adaptation of reduced metabolism in the myocytes from decreased myocardial blood flow in this early stage of CAD before manifestation of any RWMAs or reduction in EF.

Global WW was also increased, but this did not reach statistical significance. WW can occur because of systolic lengthening during LV ejection and/or myocardial shortening during isovolumic relaxation, with these contractions not contributing to LV ejection. In a normal heart, there are minimal differences in the timing of contraction of individual segments (minimal WW), but as the myocardium becomes diseased, dyssynchronous contractions and postsystolic shortening contribute to a less efficient ejection (increased WW). Overall, efficiency of the myocardium in generating "work" by longitudinal myocardial deformation is reduced.

Alternative approaches have been explored to enhance the ability of resting echocardiography to identify patients with CAD. Mor-Avi et al. $^{11}$ tested the feasibility of fusing resting three-dimensional echocardiography-derived regional LV longitudinal strain with coronary arteries from cardiac computed tomography. This allowed assessment of the hemodynamic significance of coronary stenosis in patients with chest pain without performing stress testing. This study expanded on a pilot study that found that resting strain abnormalities are more common in the presence of stenosis $>50 \%$ and perfusion defects. Fusion of cardiac computed tomography and threedimensional regional strain allowed visualization of each coronary artery and myocardial strain in its territory. The investigators found that combining computed tomographic functional flow reserve with vasodilator stress subendocardial perfusion resulted in the highest sensitivity $(83 \%)$ and specificity $(81 \%)$ to predict patients with either stenosis $>50 \%$ or perfusion defects. ${ }^{11}$

\section{Limitations}

Arrhythmias, with significant beat-to-beat variability, including atrial fibrillation, inhibit accurate and reliable assessment of GLS by STE with the viability of MW estimation questionable in such patients. Patients with left bundle branch block and pacing were also excluded from the study cohort. Patients with inadequate image quality limit the accuracy of STE, leading to suboptimal MW assessment Noninvasive systolic blood pressure cuff measurements are less accurate than invasive LV pressure measurements, but our study has already excluded any patients with aortic stenosis, LVOT obstruction, and any other cardiac pathologies that will induce a pressure gradient between aorta and LV.

The low specificity of global MW to predict CAD is a limitation of the present study. The specificity is far from optimal, which underscores the fact that global MW should not be used in isolation but in conjunction with other parameters to identify patients with significant CAD, otherwise there may be a significant number of falsepositive diagnoses. The sensitivity and specificity were also calculated in the same groups of patients in which an optimal cutoff value was made. It would be ideal to determine this cutoff value and retest in a validation population. Larger scale studies are needed for further evaluation of this new parameter to establish its clinical utility and prognostic implications. 
The distribution of CAD and the observed value in the segments that are supposed to be hypovascularized versus the segments that are supposed to vascularized normally were not assessed, because of the lack of sufficient number of patients with single lesions in each of the coronary territories (left anterior descending coronary artery, $n=18$; right coronary artery, $n=8$; circumflex coronary artery, $n=4)$. The majority of the study population $(n=50)$ had multivessel CAD, and therefore this study was not sufficiently powered for regional MW assessment.

This novel method may have potential value in clinical use in the diagnosis of early CAD at rest without the need to undergo stress imaging, but further prospective trials are required to compare MW directly with stress echocardiography, which is beyond the scope of this study.

\section{CONCLUSION}

Noninvasive LV-pressure strain loops are a novel method using STE to measure global MW, CW, WW, and GWE and account for systolic blood pressure and LV afterload. Global MW at rest is more sensitive than GLS in the detection of significant CAD ( $>70 \%$ stenosis) in patients with no RWMAs and normal EFs. This is a potential valuable clinical tool to assist in the early diagnosis of CAD.

\section{REFERENCES}

1. Elhendy A, van Domburg RT, Bax JJ, Roelandt JR. Significance of resting wall motion abnormalities in 2-dimensional echocardiography in patients without previous myocardial infarction referred for pharmacologic stress testing. J Am Soc Echocardiogr 2000;13:1-8.

2. Choi I, Cho S, Song Y, Cho S, Song B, Lee S, et al. Longitudinal 2D strain at rest predicts the presence of left main and three vessel coronary artery dis ease in patients without regional wall motion abnormality. Eur J Echocardiogr 2009; 10:695-701.

3. Galli E, Lancellotti P, Sengupta PP, Donal E. LV mechanics in mitral and aortic valve disease: value of function assessment beyond ejection fraction JACC Cardiovasc Imaging 2014;7:1151-66.

4. Montgomery D, Puthumana J, Fox J, Ogunyankin K. Global longitudinal strain aids the detection of non-obstructive coronary artery disease in the resting echocardiogram. Eur J Echocardiogr 2012;13:579-87.

5. Li L, Zhang P, Ran H, Dong J, Fang L, Ding Q. Evaluation of left ventricular myocardial mechanics by three-dimensional speckle tracking echocardiography in the patients with different gradient coronary artery stenosis. Int J Cardiovasc Imaging 2017;33:1513-20.

6. Chan J, Shiino K, Obonyo NG, Hanna J, Chamberlain R, Small A, et al. Left ventricular global strain analysis by two-dimensional speckle-tracking echocardiography: the learning curve. J Am Soc Echocardiogr 2017;30: 1081-90.

7. Luis SA, Yamada A, Khandheria BJ, Speranza V, Benjamin A, Ischenko M, et al. Use of three-dimensional speckle-tracking echocardiography for quantitative assessment of global left ventricular function: a comparative study to three-dimensional echocardiography. J Am Soc Echocardiogr 2014;27:285-91.

8. Mignot A, Donal E, Zaroui A, Reant P, Salem A, Hamon C, et al. Global longitudinal strain as a major predictor of cardiac events in patients with depressed left ventricular function: a multicentre study. J Am Soc Echocar diogr 2010;23:1019-24.

9. Cho GY, Marwick TH, Kim HS, Kim MK, Hong KS, Oh DJ. Global 2 dimensional strain as a new prognosticator in patients with heart failure. J Am Coll Cardiol 2009;54:618-24.

10. Reisner SA, Lysyansky P, Agmon Y, Mutlak D, Lessick J, Friedman Z Global longitudinal strain: a novel index of left ventricular systolic func tion. J Am Soc Echocardiogr 2004;17:630-3.
11. Mor-Avi V, Patel MB, Maffessanti F, Singh A, Medvedofsky D, Zaidi SJ, et al. Fusion of three-dimensional echocardiographic regional myocardial strain with cardiac computed tomography for noninvasive evaluation of the hemodynamic impact of coronary stenosis in patients with chest pain. J Am Soc Echocardiogr 2018;31:664-73.

12. Hubert A, Le Rolle V, Leclercq C, Galli E, Samset E, Casset C, et al. Estimation of myocardial work from pressure-strain loops analysis: an exper imental evaluation. Eur Heart J Cardiovascular Imaging 2018;19:1372-9.

13. Yingchoncharoen T, Agarwal S, Popovic Z, Markwick T. Normal ranges of left ventricular strain: a meta-analysis. J Am Soc Echocardiogr 2013;26: 185-91.

14. Skulstad H, Edvardsen T, Urheim S, Rabben SI, Stugaard M, Lyseggen E, et al. Postsystolic shortening in ischemic myocardium: active contraction or passive recoil? Circ 2002;106:718-24.

15. McCrary J, Wann LS, Thompson RC. PET imaging with FDG to guide revascularization in patients with systolic heart failure. Egypt Heart J 2013;65:123-9.

16. Donal E, Bergerot C, Thibault H, Ernande L, Loufoua J, Augeul L, et al. Influence of afterload on left ventricular radial and longitudinal systolic func tions: a two-dimensional strain imaging study. Eur J Echocardiogr 2009; 10 914-21.

17. Burns AT, La Gerche A, D’hooge J, Maclsaac A, Prior DL. Left ventricular strain and strain rate: characterization of the effect of load in human subjects. Eur J Echocardiogr 2010;11:283-9.

18. Boe E, Russell K, Eek C, Eriksen M, Remme EW, Smiseth OA, et al. Non invasive myocardial work index identifies acute coronary occlusion in patients with non-ST-segment elevation-acute coronary syndrome. Eur Heart J Cardiovasc Imaging 2015;16:1247-55.

19. Galli E, Leclercq C, Fournet M, Hubert A, Bernard A, Smiseth OA. Value of myocardial work estimation in the prediction of response to cardiac re synchronization therapy. J Am Soc Echocardiogr 2018;31:220-30.

20. Vecera J, Penicka M, Eriksen M, Russell K, Bartunek J, Vanderheydden M, et al. Wasted septal work in left ventricular dyssynchrony: a novel principle to predict response to cardiac resynchronization therapy. Eur Heart J Cardiovasc Imaging 2016;17:624-32.

21. Russell K, Eriksen M, Aaberge L, Wilhelmsen N, Skulstad H, Remme EW, et al. A novel clinical method for quantification of regional left ventricular pressure-strain loop area: a non-invasive index of myocardial work. Eur Heart J 2012;33:724-33.

22. Chan I, Edwards NFA, Khandheria BJ, Shiino K, Sabapathy S, Anderson BA, et al. A new approach to assess myocardial work by non-invasive left ventricular pressure-strain relations in hypertension and dilated cardiomyopathy. Eur Heart J Cardiovasc Imaging 2019;20: 31-9.

23. Lang RM, Badano LP, Mor-Avi V, Afilalo J, Armstrong A, Ernande L. Recommendations for cardiac chamber quantification by echocardiography in adults: an update from the American Society of Echocardiography and the European Association of Cardiovascular Imaging. J Am Soc Echocardiogr 2015;28:1-39.

24. Nagueh SF, Smiseth OA, Appleton CP, Byrd BF, Dokainish $\mathrm{H}$ Edvardsen T, et al. Recommendations for the evaluation of left ventricular diastolic function by echocardiography: an update from the American Society of Echocardiography and the European Association of Cardiovascular Imaging. J Am Soc Echocardiogr 2016;29:277-314.

25. Russell K, Eriksen M, Aaberge L, Wilhelmsen N, Skulstad H, Gjesdal O, et al. Assessment of wasted myocardial work: a novel method to quantify energy loss due to uncoordinated left ventricular contractions. Am J Physiol Heart Circ Physiol 2013;305:H996-1003.

26. Shah R, Yow E, Jones WS, Kohl LP, Kosinski AS, Hoffmann U, et al. Comparison of visual assessment of coronary stenosis with independent quantitative coronary angiography: findings from the Prospective Multicenter Imaging Study for Evaluation of Chest Pain (PROMISE) trial. Am Heart J 2016;184:1-9.

27. Patel MR, Peterson ED, Dai D, Brennan JM, Redberg RF, Anderson HV et al. Low diagnostic yield of elective coronary angiography. $N$ Engl J Med 2010;362:886-95. 
28. Norum IB, Ruddox V, Edvarsen T, Otterstad JE. Diagnostic accuracy of left ventricular longitudinal function by speckle tracking echocardiography to predict significant coronary artery stenosis. A systematic review. BMC Med Imaging 2015;15:25-37.

29. Tsai W, Liu Y, Huang Y, Lin C, Lee C, Tsai L. Diagnostic value of segmental longitudinal strain by automated function imaging in coronary artery disease without left ventricular dysfunction. J Am Soc Echocardiogr 2010;23: 1183-9.

30. Dahlslett T, Karlsen S, Grenne B, Eek C, Sjoli B, Skulstad H, et al. Early assessment of strain echocardiography can accurately exclude significant coronary artery stenosis in suspected non-ST-segment elevation acute coronary syndrome. J Am Soc Echocardiogr 2014;27:512-9.

31. Sjoli B, Orn S, Grenne B, Ihlen H, Edwardsen T, Brunvand H. Diagnostic capability and reproducibility of strain by Doppler and by speckle tracking in patients with acute myocardial infarction. JACC Cardiovasc Imaging 2009;2:24-33.

32. Shimoni S, Gendelman G, Ayzenberg O, Smirin N, Lysyansky P, Edri O, et al. Differential effects of coronary artery stenosis on myocardial function: the value of myocardial strain analysis for the detection of coronary artery disease. J Am Soc Echocardiogr 2011;24:748-57.

33. Winter R, Jussila R, Nowak J, Brodin LA. Speckle tracking Echocardiography is a sensitive tool for the detection of myocardial ischemia: a pilot study from the catheterization laboratory during percutaneous coronary intervention. J Am Soc Echocardiogr 2007;20:974-81.

34. Reimer K, Jennings R. The "wavefront phenomenon" of myocardial ischemic cell death. II. Transmural progression of necrosis within the framework of ischemic bed size (myocardium at risk) and collateral flow. Lab Invest 1979;40:633-44.

35. Hanekom L, Cho GY, Leano R, Jeffriess L, Marwick TH. Comparison of two-dimensional speckle and tissue Doppler strain measurement during dobutamine stress echocardiography: an angiographic correlation. Eur Heart J 2007;28:1765-72.

36. Gaibazzi N, Pigazzani F, Reverberi C, Porter Thomas R. Rest global longitudinal 2D strain to detect coronary artery disease in patients undergoing stress echocardiography: a comparison with wall-motion and coronary flow reserve responses. Echo Res Pract 2014;1:61-70.

37. Evensen K, Sarvari S, Rønning OM, Edvardsen T, Russell D. Carotid artery intima-media thickness is closely related to impaired left ventricular function in patients with coronary artery disease: a singlecentre, blinded, non-randomized study. Cardiovas Ultrasound 2014; 12:39-46.

38. Biering-Sørensen T, Hoffman S, Mogelvang R, Iversen AZ, Galatius S, Fritz-Hansen T, et al. Myocardial strain analysis by 2-dimensional speckle tracking echocardiography improves diagnostics of coronary artery stenosis in stable angina pectoris. Circ Cardiovasc Imag 2014;7:58-65.

39. Ghosh N, Rimoldi OE, Beanlands SB, Camici PG. Assessment of myocardial ischaemic and viability: role of positron emission tomography. Eur Heart J 2010;31:2984-95.

40. Aoyama R, Takano H, Kobayashi Y, Kitamura M, Asai K, Amano Y, et al. Evaluation of myocardial glucose metabolism in hypertrophic cardiomyopathy using ${ }^{18} \mathrm{~F}$-fluorodeoxyglucose positron emission tomography. PLoS One 2017;12:e0188479. 